\title{
Prevalence of malaria and scrub typhus co-infection in febrile patients: a systematic review and meta-analysis
}

\author{
Polrat Wilairatana ${ }^{1}$, Saruda Kuraeiad ${ }^{2}$, Pongruj Rattaprasert ${ }^{3}$ and Manas Kotepui ${ }^{*}$
}

\begin{abstract}
Background: Little information is available about malaria and scrub typhus co-infection. This study aimed to investigate the pooled prevalence of malaria and scrub typhus co-infection in febrile patients. Further, it aimed to estimate the prevalence of scrub typhus infection among patients with malaria and the odds of co-infection. This will aid the diagnosis and management of co-infected patients in endemic areas.

Methods: We searched for relevant studies in three databases: PubMed, Scopus, and Web of Science. We assessed the quality of the included studies using the Joanna Briggs Institute checklist for analytical cross-sectional studies. We estimated (1) the pooled prevalence of malaria and scrub typhus co-infection, (2) the pooled prevalence of scrub typhus infection in malaria-positive patients, and (3) the pooled odds of co-infection using the DerSimonian-Laird method for random-effects models. The study results and summary estimates were visualized on a forest plot as point estimates (effect size, prevalence) and 95\% confidence intervals (Cl). We assessed the heterogeneity of the studies by Cochrane Q or $P$ statistics. We performed subgroup analyses of countries and scrub typhus diagnostic tests to explore the sources of heterogeneity of the included studies. We assessed publication bias if more than 10 studies were used to estimate the outcome. All data analyses were conducted using Stata version 14 (StataCorp, College Station, TX, USA).

Results: Of the 542 studies retrieved from three databases, we included 14 meeting the inclusion criteria in the systematic review and meta-analysis. The pooled prevalence of malaria and scrub typhus co-infection (56 cases) among febrile patients (7920 cases) was 1\% (95\% Cl: 0-1\%, P: 78.28\%), while the pooled prevalence of scrub typhus infection (321 cases) in patients with malaria (1418 cases) was 21\% (95\% Cl: 12-30\%, $\left.I^{2}: 98.15 \%\right)$. Subgroup analysis showed that the pooled prevalence of scrub typhus infection among patients with malaria in India was 8\% (95\% Cl: 4-13\%, $P: 85.87 \%$, nine studies with 59/794 cases), while the pooled prevalence of scrub typhus infection among patients with malaria in Thailand was 35\% (95\% Cl: 7-64\%, P: 98.9\%, four studies with 262/624 cases). The co-infections did not occur by chance ( $P=0.013$, odds: $\left.0.43,95 \% \mathrm{Cl}: 0.22-0.84 \%, P^{2}: 60.9 \%\right)$. In the sensitivity analysis, the pooled prevalence of malaria and scrub typhus co-infection among febrile patients was $0 \%$ (95\% Cl: 0-1\%, P: $59.91 \%)$.
\end{abstract}

Conclusions: The present study showed the pooled prevalence and a significant association between malaria and scrub typhus. The results show the status of co-infection. Further research into co-infection in endemic areas is

*Correspondence: manas.ko@wu.ac.th

2 Department of Medical Technology, School of Allied Health Sciences,

Walailak University, Tha Sala, Nakhon Si Thammarat 80160, Thailand

Full list of author information is available at the end of the article permits use, sharing, adaptation, distribution and reproduction in any medium or format, as long as you give appropriate credit to the original author(s) and the source, provide a link to the Creative Commons licence, and indicate if changes were made. The images or other third party material in this article are included in the article's Creative Commons licence, unless indicated otherwise in a credit line to the material. If material is not included in the article's Creative Commons licence and your intended use is not permitted by statutory regulation or exceeds the permitted use, you will need to obtain permission directly from the copyright holder. To view a copy of this licence, visit http://creativecommons.org/licenses/by/4.0/. The Creative Commons Public Domain Dedication waiver (http://creativeco mmons.org/publicdomain/zero/1.0/) applies to the data made available in this article, unless otherwise stated in a credit line to the data. 
needed, in particular, to determine whether co-infection can accelerate disease progression or protect against severe disease.

Keywords: Scrub typhus, Orientia tsutsugamushi, Malaria, Plasmodium spp., Co-infection

\section{Background}

Blood-feeding Anopheles mosquitoes transmit malaria by one of the six Plasmodium species, P. falciparum, $P$. vivax, $P$. malariae, $P$. ovale curtisi, $P$. ovale wallikeri, and $P$. knowlesi; however, some zoonotic Plasmodium species, such as $P$. cynomolgi and $P$. simium, have been associated with human cases of malaria [1-5]. In a high transmission area, malaria is a major cause of death in children under 5 years because they lack immunity against malaria parasites; malaria is less common in older children and adults because of partial immunity [6]. In a low transmission setting, symptomatic malaria may occur at all ages, particularly in semi-immune or non-immune people $[7,8]$. In 2020, the World Health Organization (WHO) reported that the Southeast Asian region accounted for $\sim 3 \%$ of the global malaria burden of 6.3 million, and malaria deaths reached $\sim 9000$ in 2019 [7]. Within the WHO South-East Asia Region, India had $86 \%$ of malaria deaths, while in the Greater Mekong Subregion, Cambodia (58\%) and Myanmar (31\%) reported the most malaria cases [7].

Scrub typhus, which causes an acute febrile illness, is caused by the Gram-negative obligate intracellular bacillus Orientia tsutsugamushi. It is transmitted via trombiculid mite chigger (larva) bites [9] and is associated with agricultural work and rural dwellings [10,11]. Recent findings suggest that other Orientia species are related to scrub typhus, including "Candidatus Orientia chiloensis" [12] and "Candidatus Orientia chuto" $[13,14]$. Scrub typhus is endemic to the Asian-Pacific area with a seroprevalence ranging from $9.3 \%$ to $27.9 \%$ (median 22.2\% interquartile range [IQR] 18.6-25.7). Reported case-fatality rates are $12.2 \%$ in South India and $13.6 \%$ in northern Thailand [15]. Scrub typhus seropositivity has also been evidenced in Honduras [16] and the Peruvian Amazon [17], with a seroprevalence of 5.6\% and $5.3 \%$. A recent finding reported that cases of scrub typhus had been found in southern Chile [13, 14, 18]. Therefore, scrub typhus is not limited to the Tsutsugamushi Triangle.

Clinical signs and symptoms of scrub typhus are fever, headache, myalgia, cough, and gastrointestinal symptoms, with or without escharation [19]. A recent study from northern Vietnam reported that $70 \%$ of patients with scrub typhus had eschars, $60 \%$ had skin-conjunctiva congestion, and $44 \%$ had lymphadenopathy [20]. Untreated acute scrub typhus cases have a high risk of developing severe manifestations, including microangiopathy, septic shock, acute respiratory failure, congestive heart failure, severe jaundice, or acute renal failure [19]. Furthermore, a systematic review showed high mortality in scrub typhus cases with central nervous system involvement or multi-organ dysfunction in pregnant women [15]. Reliable diagnostic tests would help physicians manage suspected scrub typhus cases.

Little information is available about malaria and scrub typhus co-infection. We aimed to investigate the pooled prevalence of malaria and scrub typhus co-infection among febrile patients, the prevalence of scrub typhus infection among patients with malaria, and the odds of co-infection. The data will help diagnose and manage coinfected patients in endemic areas.

\section{Methods}

\section{Protocol and registration}

The protocol of this systematic review was registered at PROSPERO with ID CRD42021255893. We followed the preferred reporting criteria for systematic reviews and meta-analyses (PRISMA) [21].

\section{Search strategy}

We used three databases to search for relevant studies, PubMed, Scopus, and Web of Science. We used the keyword combination "(malaria OR plasmodium) AND scrub typhus." The date range was from inception to May 12, 2021. The searches were unrestricted for language or year of publication. We also searched reference lists and Google Scholar to maximize studies and prevent relevant studies from being missed. The search strategy is described fully in Additional file 1: Table S1.

\section{Eligibility criteria}

We used the PICo (P: participants, I: phenomena of interest, Co: context) or PICO (P: participants, I: phenomena of interest, C: control, O: outcome of interest) approach to select eligible studies. For the primary outcome, we used (i) P: febrile patients suspected of malaria or scrub typhus infection, (ii) I: patients with co-infection with malaria and scrub typhus, (iii) Co: none. For the secondary outcome, we used (i) P: patients with malaria infection, (ii) I: patients with co-infection of malaria and scrub typhus, (iii) Co: none. For the tertiary outcome, we used (i) P: febrile patients suspected of malaria or scrub typhus infection, (ii) I: patients with co-infection of malaria and 
scrub typhus, (iii) C: febrile patients without malaria and scrub typhus, and (iv) O: chance of co-infection. We detected Plasmodium infections by microscopy (gold standard), rapid diagnostic test (RDT), or a molecular method. We detected scrub typhus infections using indirect fluorescence assay (IFA) (gold standard), IgM enzyme-linked immunosorbent assay (ELISA), or RDT. All studies that reported co-infections with malaria and scrub typhus were considered. Case reports and case series, reviews, correspondence, animal studies, experimental studies, and test evaluation studies were excluded.

\section{Study selection}

We reviewed all studies reporting malaria and scrub typhus co-infection. First, we excluded duplicates. Second, we screened the titles and abstracts, excluding nonrelated studies. Third, we read the full text and excluded irrelevant studies for various reasons. The study selection process was performed using EndNote X8 for reference management. Two authors (MK, SK) selected the studies independently and resolved any disagreements by a discussion with the third author (PW).

\section{Data extraction}

We extracted the following information: first author's name, year of publication, study site, year conducted, study design, age group, gender, number of participants, number of co-infections, number of malaria cases, number of scrub typhus cases, diagnostic test for malaria, and diagnostic test for scrub typhus. We recorded the data in a spreadsheet for further analysis. Two authors (MK and SK) independently extracted the data and resolved any inconsistency or disagreement by consensus.

\section{Quality of the included studies}

We assessed the quality of the selected studies using the checklist for analytical cross-sectional studies developed by the Joanna Briggs Institute. The checklist was based on study design, study conduct, and analysis of the outcome of interest. High-quality studies received seven to eight points, while moderate-quality studies scored four to six points. Studies scoring fewer than four points were excluded. Two authors (MK and SK) assessed the included studies' quality.

\section{Data analysis}

The pooled prevalence of malaria and scrub typhus coinfection among febrile patients was estimated using the DerSimonian-Laird method for the random-effects model, based on the inverse variance approach for measuring weight as described previously $[22,23]$. The number of co-infected patients and participants tested for both pathogens was used in the meta-analysis. The pooled prevalence of scrub typhus infection among malaria-positive patients was estimated by applying the random-effect model using the number of scrub typhus infections and malaria-positive cases. The pooled odds of co-infection were estimated using the following data: (1) the number of co-infections, (2) the number of malaria infections without scrub typhus, (3) the number of scrub typhus infections without malaria, and (4) the number of febrile patients without scrub typhus or malaria. The individual study results and the summary estimates were visualized with a forest plot as point estimates (effect size, prevalence) and $95 \%$ confidence intervals (CI). The heterogeneity of the included studies was assessed by Cochrane Q $(P>0.05$ indicated significant heterogeneity) or $I^{2}$ statistics, with $I^{2}$ values $<25 \%, 25-75 \%$, and $>75 \%$ interpreted as low, moderate, and high heterogeneity, respectively [24]. We analyzed the subgroup analysis of countries and diagnostic tests for scrub typhus to explore the source of heterogeneity among the included studies. Publication bias was assessed if more than 10 studies were used to estimate the outcome [25]. All data analyses were performed using Stata version 14 (StataCorp, College Station, TX, USA).

\section{Results}

\section{Search results}

Among the 542 studies retrieved from the three databases, 10 studies [26-35] met the inclusion criteria. We found four studies [36-39] in the reference lists of the included studies and Google Scholar. Finally, 14 studies were included in this systematic review and meta-analysis (Fig. 1).

\section{Characteristics of the included studies}

Fourteen studies that reported malaria and scrub typhus co-infections were included in the study (Table 1). All the studies were published between 1998 and 2020. Six studies $(42.9 \%)$ were retrospective observational studies [26, 30, 33, 34, 36, 38], six were cross-sectional studies [27-29, 31, 37, 39], and two [32, 35] were cohort studies. Most studies $(10 / 14,71.4 \%)$ were conducted in India [26-28, 30-33, 37-39], and four studies [29, 34-36] were conducted in Thailand. Nine studies [26-28, 31-33, 35, $38,39]$ enrolled febrile patients (7920 cases), four [29, 34, 36,37 ] enrolled malaria-positive patients (639 cases), and one study [30] enrolled scrub-typhus-positive patients (240 cases). In the febrile patient studies, 56 co-infections in 7920 patients were reported, and in the studies that enrolled malaria-positive patients [29, 34, 36, 37], 265 of 639 cases were reported co-infected. Six concurrent malaria infections were reported in the study [30] that enrolled 240 scrub-typhus-positive patients. The enrolled patients were $>12$ years old in seven studies $[29,30,32$, 


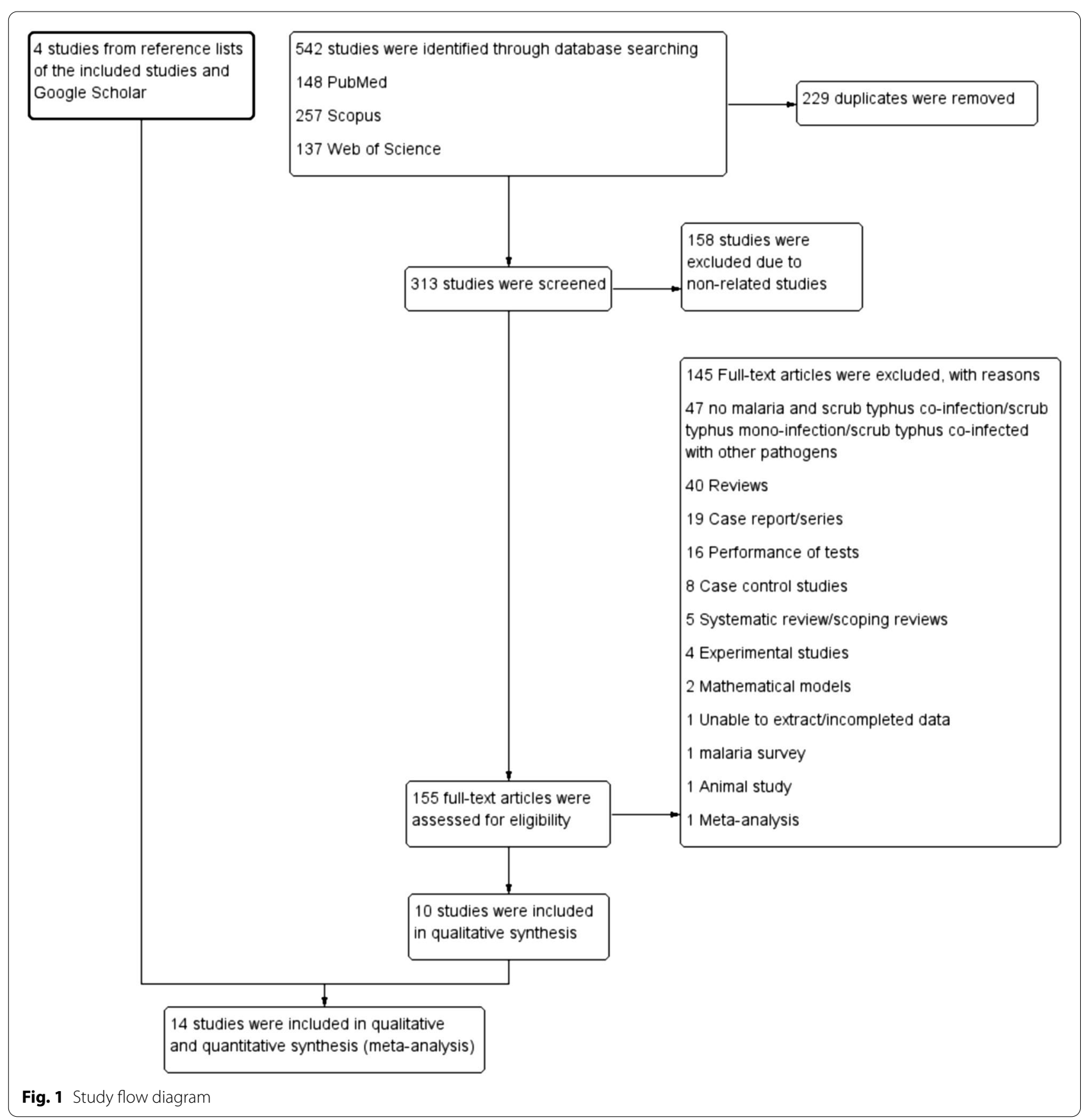




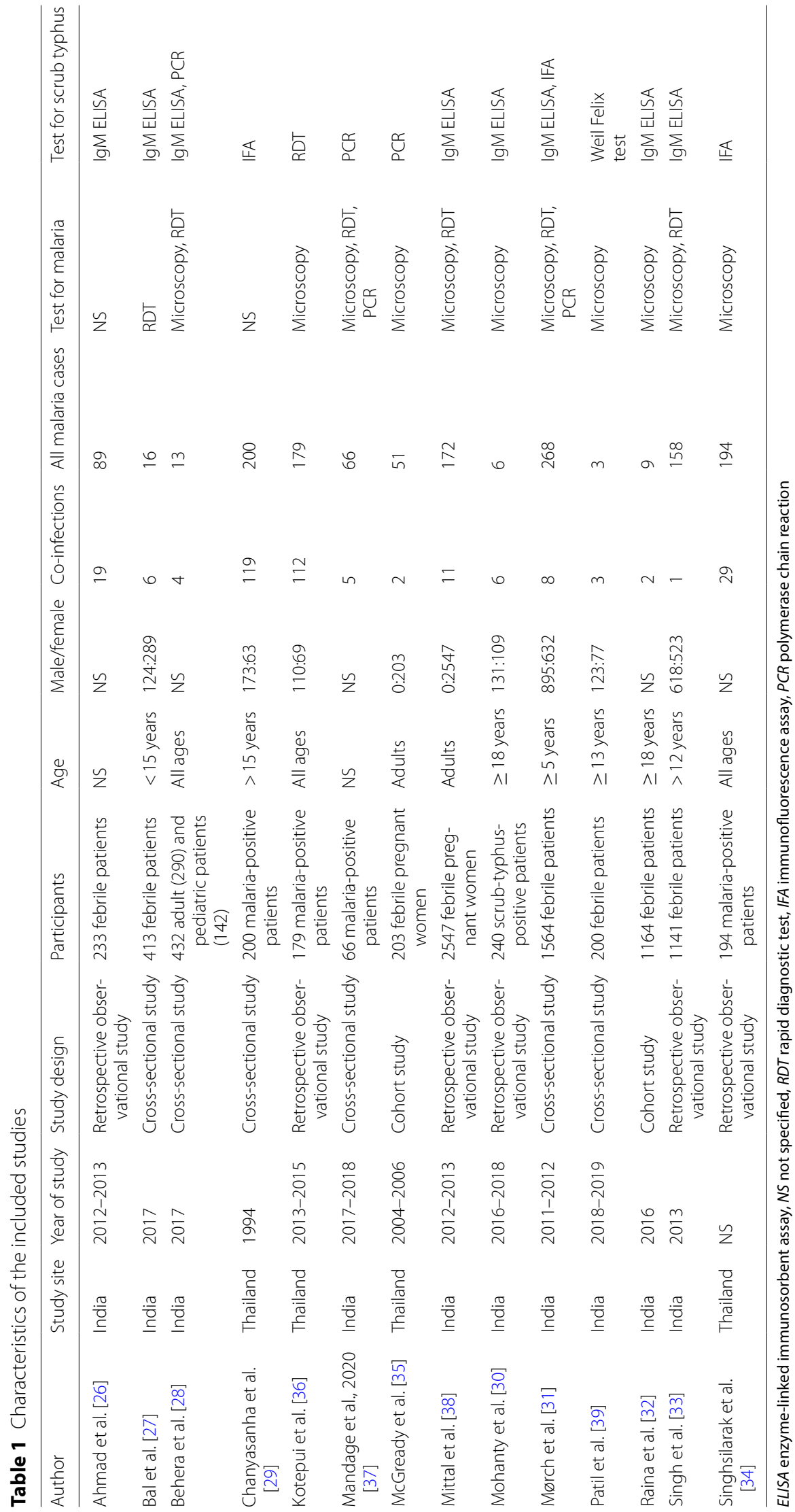


$33,35,38,39]$, any age groups in two [29-33], and ages were not specified (NS) in two [26, 37].

\section{Quality of the included studies}

The quality of the included studies was assessed using the Joanna Briggs Institute checklist for analytical crosssectional studies. Four studies [29, 31, 35, 38] were considered high-quality studies, while 10 studies [26-28, 30, $32-34,36,37,39]$ were categorized as moderate-quality studies (Additional file 2: Table S2).

\section{Prevalence of malaria and scrub typhus co-infection among febrile patients}

The pooled prevalence of malaria and scrub typhus coinfection (56 cases) among febrile patients (7920 cases) was estimated from nine studies [26-28, 31-33, 35, 38, 39]. The highest prevalence of co-infection was demonstrated in a study by Ahmad et al. (8\%) [26], while the lowest prevalence $(0 \%)$ was seen in three studies [32, 33, 38]. Overall, the results showed that the pooled prevalence of malaria and scrub typhus co-infection in febrile patients was $1 \%$ (95\% CI: $0-1 \%, I^{2}: 78.28 \%$ ) (Fig. 2).

\section{Prevalence of scrub typhus infections among patients with malaria}

The pooled prevalence of scrub typhus infection, 321 out of 1418 patients with malaria (cases), was estimated from
13 studies [26-29, 31-39]. In India, the results of individual studies showed prevalence heterogeneity (1-100\%); the highest prevalence (100\%) was reported in the study by Patil et al. [39], while the lowest prevalence was found by Singh et al. [33] (1\%, 95\% CI: 0-3\%). Overall, the results showed that the pooled prevalence of scrub typhus infection among patients with malaria in India was $8 \%(95 \%$ CI: $4-13 \%, I^{2}: 85.87 \%$, nine studies with $59 / 794$ cases).

In Thailand, studies also showed prevalence heterogeneity (4-63\%), in which the studies by Kotepui et al. [36] and Chanyasanha et al. [29] demonstrated high prevalence at 63\% (95\% CI: 55-69\%) and 60\% (95\% CI: 53-66\%), respectively, while the lowest prevalence was demonstrated by McGready et al. [35], with 4\% (95\% CI: $1-13 \%)$. Overall, the pooled prevalence of scrub typhus infection in patients with malaria in Thailand was $35 \%$ (95\% CI: 7-64\%, $I^{2}$ : 98.9\%, four studies with $262 / 624$ cases). Combined with the results of the estimated prevalence in India and Thailand, the pooled prevalence of scrub typhus infection among patients with malaria was 21\% (95\% CI: 12-30\%, $I^{2}$ : 98.15\%) (Fig. 3).

\section{Subgroup analysis by diagnostic tests}

The subgroup analysis of the diagnostic tests for scrub typhus was performed using 13 studies [26-29, 31-39]. The pooled prevalence of scrub typhus infection among patients diagnosed with malaria was $12 \%(95 \% \mathrm{CI}$ :

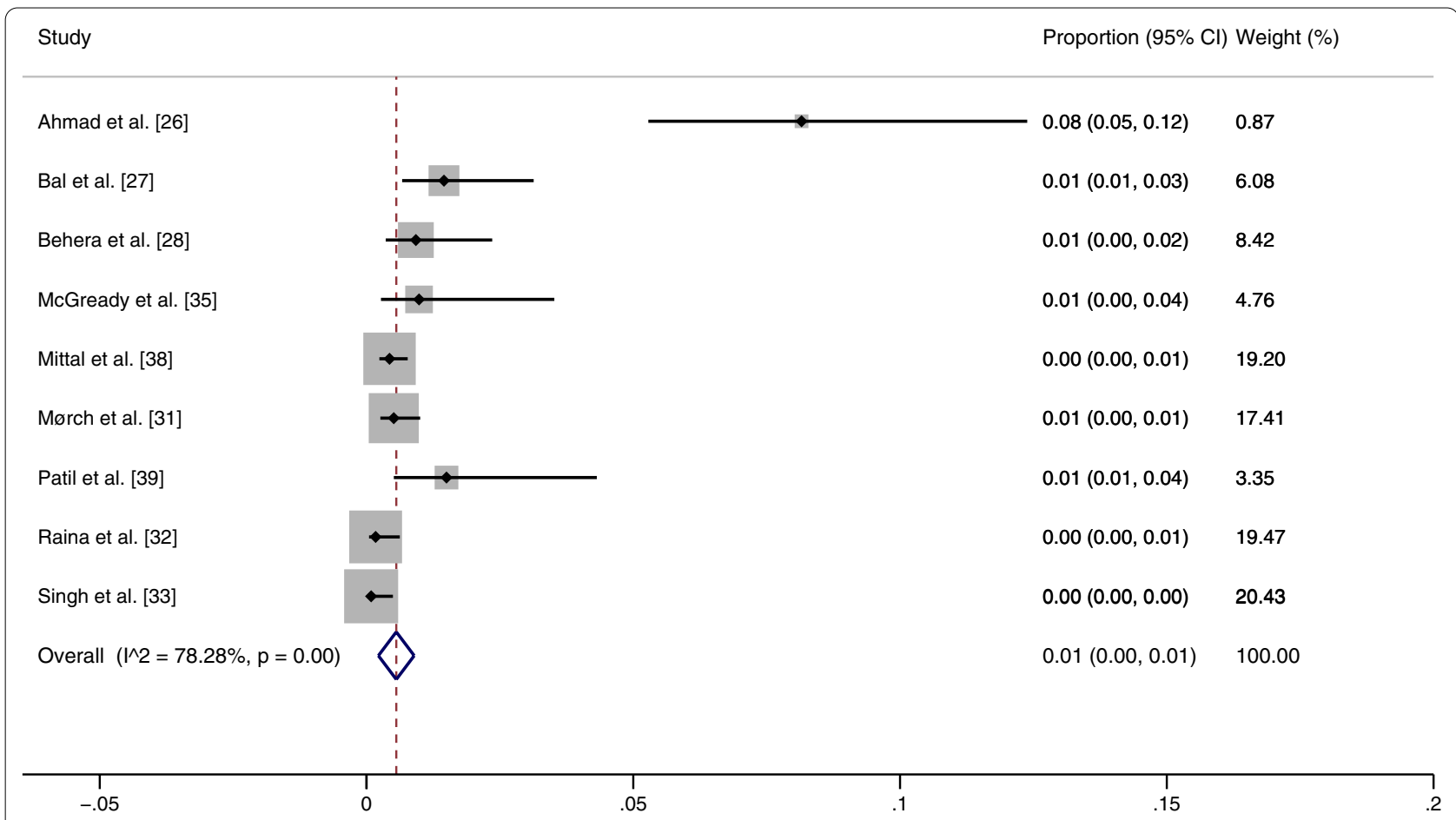

Fig. 2 Meta-analysis for pooled prevalence of malaria and scrub typhus co-infection in febrile patients. Cl confidence interval 


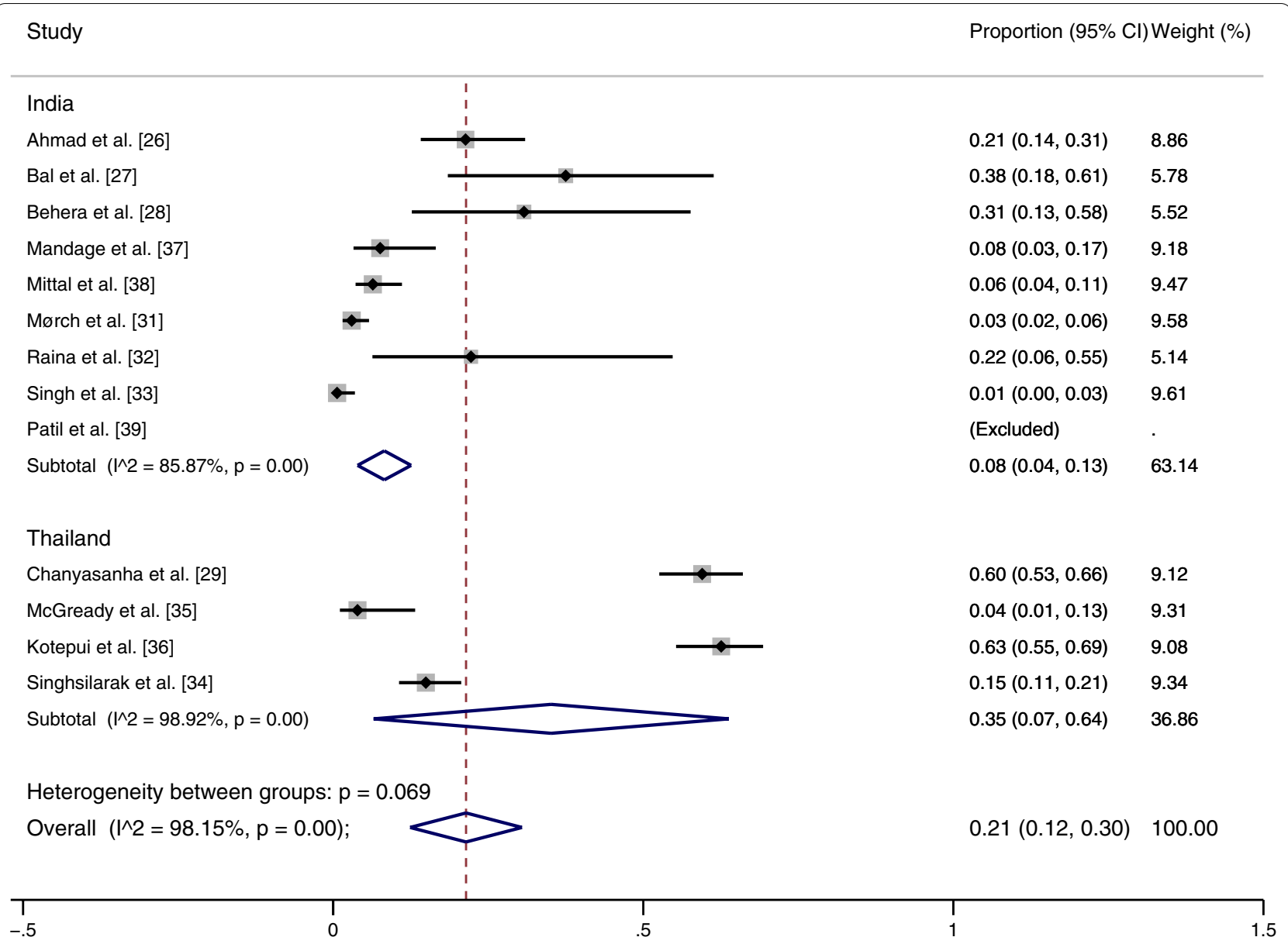

Fig. 3 Meta-analysis for pooled prevalence of scrub typhus in patients with malaria according to country (India and Thailand). Cl confidence interval

4-20\%, $\left.I^{2}: 90.15 \%\right)$ according to IgM ELISA alone, 31\% with IgM ELISA/polymerase chain reaction (PCR) $(95 \%$ CI: $13-58 \%), 31 \%$ with IFA alone (95\% CI: $27-35 \%, I^{2}$ : 97\%), 3\% with IgM ELISA/IFA (95\% CI: 2-6\%), 5\% with PCR (95\% CI: $1-10 \%), 63 \%$ with RDT (95\% CI: 55-69\%), and $100 \%$ with the Weil Felix test (Fig. 4).

\section{Odds of co-infection}

The pooled odds of co-infection were estimated using seven studies [26-28, 31, 32, 35, 38]. Three studies [26, 31,38 ] demonstrated lower odds of co-infection, but four studies $[27,28,32,35]$ showed no difference in the odds of co-infection. Overall, the results showed that malaria and scrub typhus co-infection did not occur by chance $\left(P=0.001\right.$, odds: $0.41,95 \%$ CI: $\left.0.24-0.68 \%, I^{2}: 53.9 \%\right)$ (Fig. 5).

\section{Sensitivity analysis}

Because the pooled prevalence of malaria and scrub typhus co-infection among febrile patients was heterogeneous, we used the leave-one-out method [40] for statistical validity and homogeneity of the results. Ahmad et al's [26] study in India, with the highest prevalence, was excluded as an outlier. The sensitivity analysis showed that the pooled prevalence of malaria and scrub typhus co-infection among febrile patients was very low (0\%, 95\% CI: 0-1\%, $I^{2}: 59.91 \%$ ) (Fig. 6). The fixed-effect model showed that the pooled prevalence of malaria and scrub typhus co-infection among febrile patients was $0 \%$ (95\% CI: $\left.0-0 \%, I^{2}: 0 \%\right)$ in India and 1\% (95\% CI: 0-4\%, $I^{2}$ : $0 \%$ ) in Thailand (Fig. 7).

\section{Publication bias}

Publication bias was assessed by a funnel plot using the effect size (ES, pooled prevalence) and standard error of the ES (seES) from the 12 studies [26-29, 31-38] that assessed the pooled prevalence of scrub typhus infection in patients with malaria. The results showed the asymmetric distribution of the ES (Fig. 8a). The Egger's test showed that small study effects occurred $(P=0.023$, 


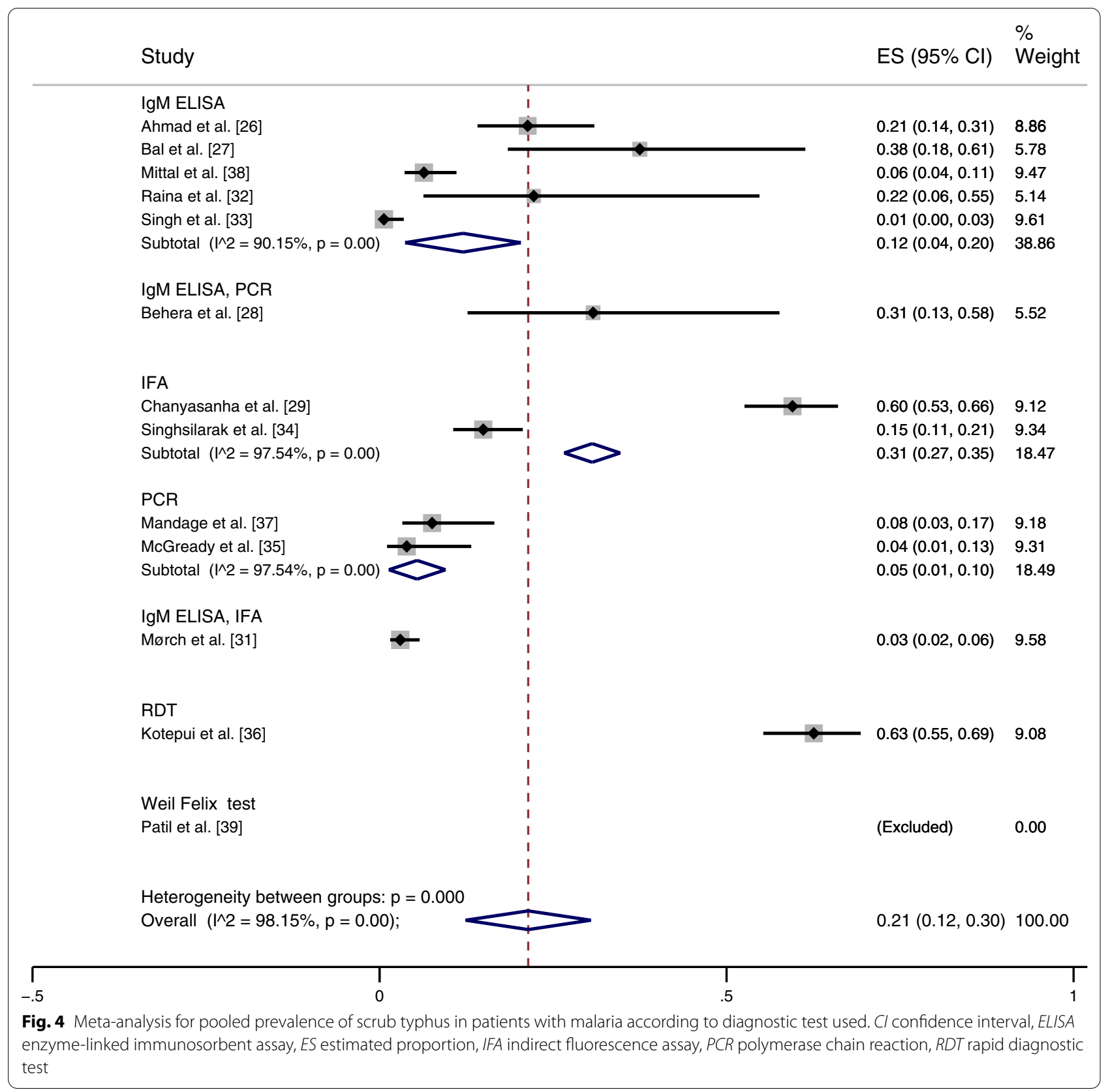

coefficient: 6.55, standard error: 2.43, $t: 2.69$ ). Further contour-enhanced funnel plot analysis showed that most of the studies missing from non-significant areas $(P>0.01)$ indicated that the cause of funnel plot asymmetry was likely to be due to publication bias (Fig. 8b).

\section{Discussion}

Malaria and scrub typhus are vector-borne diseases transmitted by different arthropod vectors $[41,42]$. The meta-analysis in this study showed a low prevalence $(0-1 \%)$ of malaria and scrub typhus co-infection among febrile patients, and these types of infections were limited to India. Although there was heterogeneity of prevalence in the studies included in the meta-analysis (78\%), four studies conducted in India demonstrated the same prevalence (1\%). The study by Ahmad et al. (8\%) [26], which differed, used IgM ELISA to detect scrub typhus IgM antibodies. It is unknown whether malaria infection can induce IgM antibodies that cross-react with other fevers. A report suggested that an apparent high co-infection rate might be caused by cross-reactivity and the background positivity of patients in endemic 


\begin{tabular}{|c|c|c|}
\hline Study & Proportion $(95 \% \mathrm{Cl})$ & Weight (\%) \\
\hline Ahmad et al. [26] & $0.18(0.10,0.34)$ & 20.68 \\
\hline Bal et al. [27] & $0.62(0.22,1.74)$ & 13.41 \\
\hline Behera et al. [28] & $1.25(0.38,4.14)$ & 11.30 \\
\hline McGready et al. [35] & $0.58(0.12,2.74)$ & 7.98 \\
\hline Mittal et al. [38] & $0.39(0.21,0.72)$ & 20.39 \\
\hline Mørch et al. [31] & $0.23(0.11,0.48)$ & 18.44 \\
\hline Raina et al. [32] & $0.91(0.19,4.43)$ & 7.80 \\
\hline Overall $(I-$ squared $=53.9 \%, p=0.043$ ) & $0.41(0.24,0.68)$ & 100.00 \\
\hline NOTE: Weights are from random effects analysis & & \\
\hline $\begin{array}{c}1 \\
.0998\end{array}$ & 0 & \\
\hline
\end{tabular}

Fig. 5 Meta-analysis for odds of malaria and scrub typhus co-infection in febrile patients. Cl confidence interval

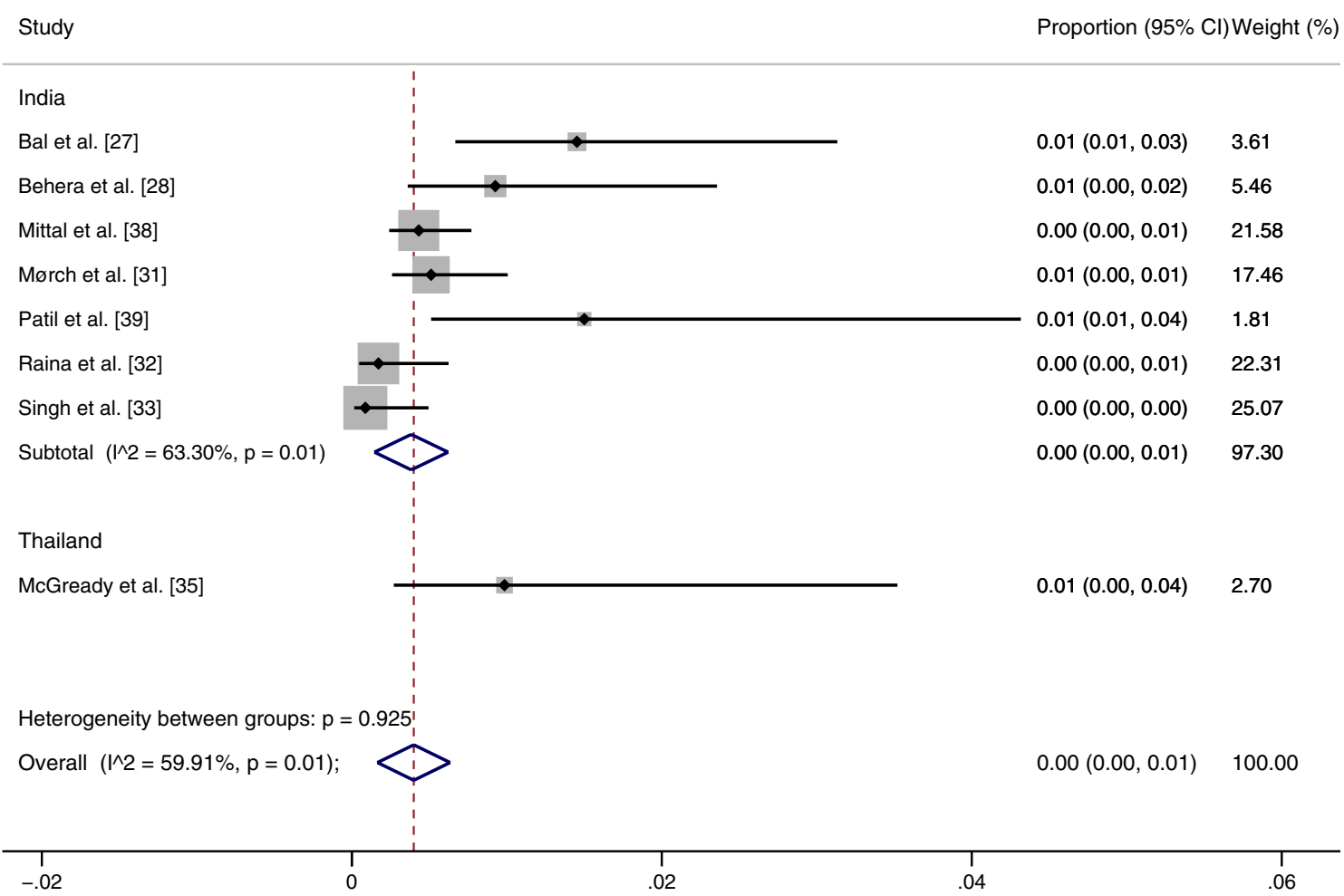

Fig. 6 Sensitivity test of the pooled prevalence of malaria and scrub typhus co-infection in febrile patients when the outlier was excluded. Cl confidence interval 


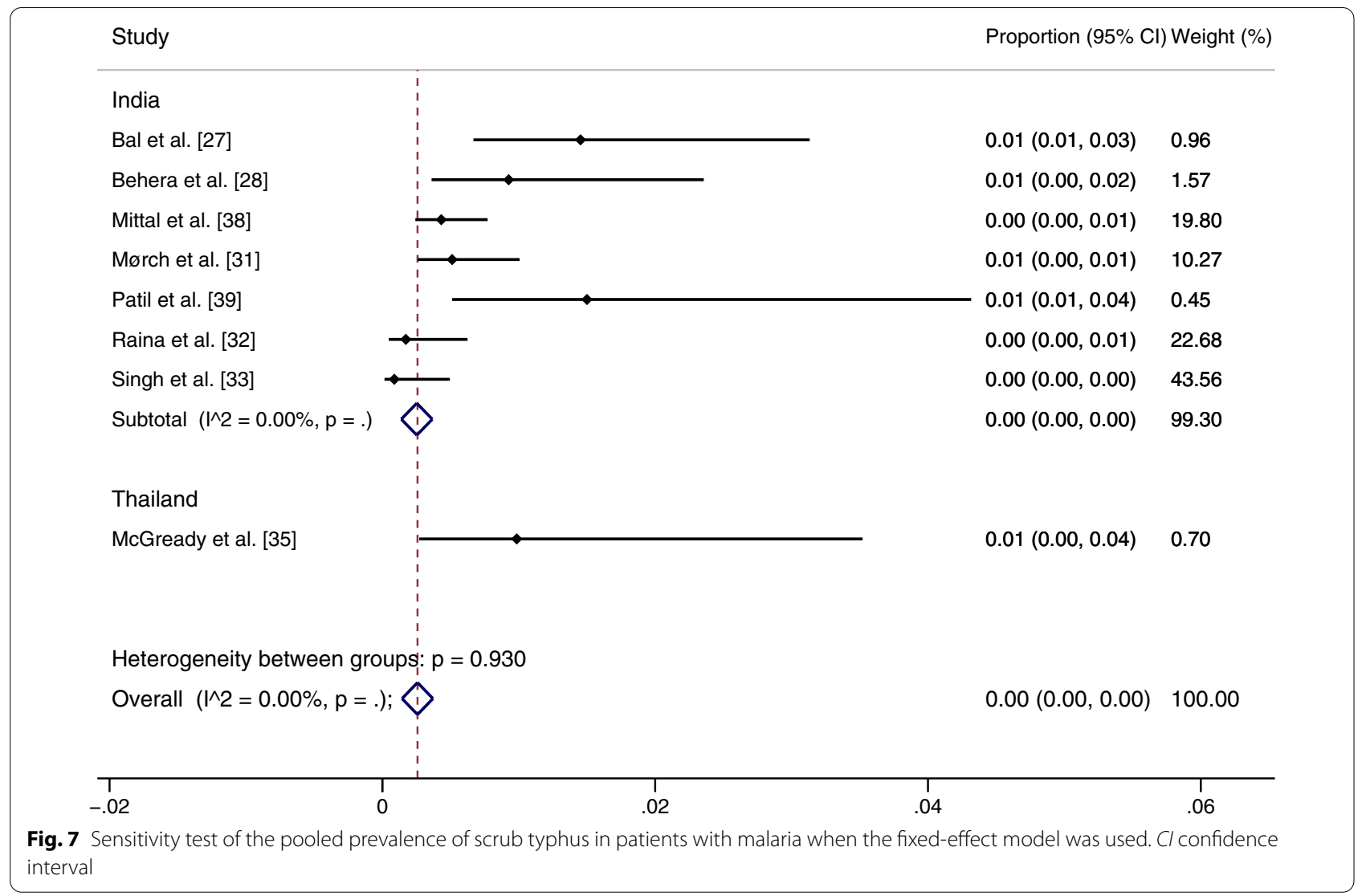
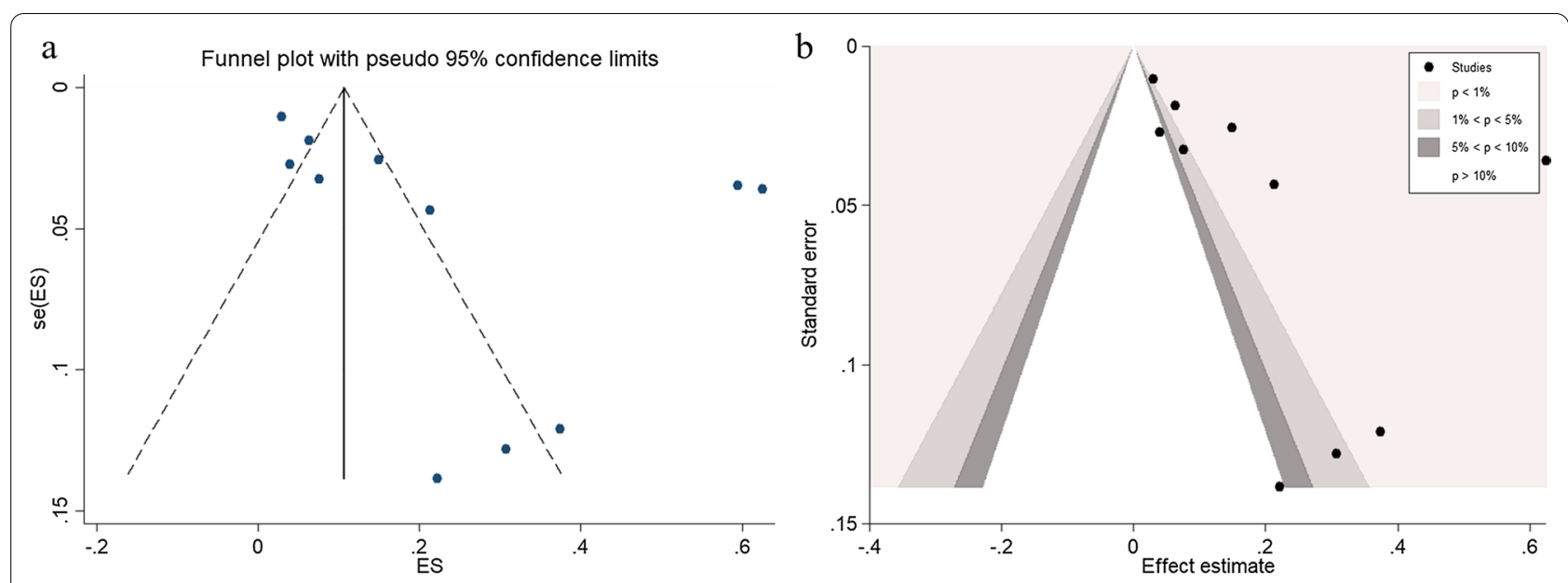

Fig. 8 Funnel plot (a) and contour-enhanced funnel plot (b)

areas rather than actual co-infection [31]. Therefore, the results of a single serological test for scrub typhus diagnosis should be interpreted cautiously if malaria is endemic in the same area. A previous study showed that malaria and scrub typhus co-infection reduced hepatosplenomegaly or other organ dysfunctions, compared with malaria and scrub typhus mono-infections. However, only small groups of patients were investigated [26]. The mechanisms of protection against severe disease are not well understood, but a recent study conducted in India showed that malaria and scrub typhus co-infection is associated with severe malaria (adjusted OR: 1.1, 95\% CI: $0.1-7.8 \%$ ) [37]. This suggests that this co-infection might be like malaria and dengue co-infection in its impact on 
disease severity, leading to severe malaria or severe dengue [43]. However, the co-infection prevalence data for these pathogens are limited. Further research is needed to determine whether co-infecting pathogens can impact disease outcomes.

Our meta-analysis showed a high prevalence of scrub typhus infections among patients with malaria in Thailand (35\%). This was associated with high heterogeneity (98.9\%), possibly caused by differences in the study participants, scrub typhus diagnostic tests, study area, or the year of the studies. In the study by Kotepui et al. [36], RDT was used to detect scrub typhus IgM/IgG. The manufacturer claims it provides $100 \%$ sensitivity, $100 \%$ specificity, and 100\% accuracy (Rickettsia IgG/IgM Combo Test, LumiQuick Diagnostics, Inc., USA). The study design involved retrospective collection of the data of patients who had been previously infected with malaria and tested for scrub typhus; therefore, the prevalence of scrub typhus infections in malaria patients may have been overestimated. Chanyasanha et al. [29] demonstrated a prevalence as high as that reported by Kotepui et al. [36]. In contrast, a study of pregnant women who attended antenatal care by Mittal et al. [38] showed a low prevalence of scrub typhus infections among patients with malaria $(6 \%)$. The difference in prevalence might be due to the high prevalence of scrub typhus before the year 2000, when, as the study by Chanyasanha et al. [29] showed, there was a prevalence of $59.5 \%$ among malaria clinic patients. After 2000, the scrub typhus prevalence was $9.1-11.1 \%$ [44]. Retrospective diagnosis with RDT showed a similar performance to ELISA in Indian patients, and the RDT diagnosis of scrub typhus was more sensitive than standard IFA in acute-phase specimens [45]. Nevertheless, a recent meta-analysis showed the pooled sensitivity and specificity of commercially available RDTs were $66.0 \%$ (95\% CI $0.37-0.86 \%$ ) and 92.0\% (95\% CI $0.83-0.97 \%$ ), respectively, with a high degree of heterogeneity between the reviewed studies [46]. The performance of RDTs makes them a good choice for the early diagnosis of scrub typhus in remote or rural regions of endemic countries.

In India, a meta-analysis demonstrated a low prevalence of malaria and scrub typhus co-infection in febrile patients and a low prevalence of scrub typhus infection in malaria patients. There was heterogeneity in the prevalence of malaria and scrub typhus co-infection in febrile patients among the studies conducted in India $(0-1 \%)$. However, the prevalence of co-infection tended toward homogeneity when the meta-analysis excluded the results of Ahmad et al. [26]. The low prevalence of malaria and scrub typhus co-infection among febrile patients might be because it is understudied or underreported, as suggested by Mørch et al. [31]. Epidemiological studies have reported that socioeconomic status and occupation were related to the risk of scrub typhus $[47,48]$. Scrub typhus prevalence is $\sim 3-9 \%$ in Vietnam $[49,50], 6-23 \%$ in Bhutan [51, 52], and $4-5 \%$ in Malaysia [53, 54]. In addition, scrub typhus was reported in China, Japan, South Korea, Indonesia, the Philippines, and Australia [55, 56]. However, malaria and scrub typhus co-infections only occurred in Thailand and India; the reasons for this are poorly understood. Possibly, physicians in countries other than Thailand and India did not investigate febrile patients for scrub typhus, as there is no Global Fund support for this, unlike with acquired immunodeficiency syndrome, tuberculosis, and malaria [57]. Also, some febrile patients may self-medicate with drugs like doxycycline, which is an anti-rickettsia drug and can lead to the misdiagnosis of scrub typhus. Therefore, the underreporting of scrub typhus in febrile or malaria patients is likely.

The present meta-analysis showed significantly decreased odds of co-infection, which indicates that malaria and scrub typhus co-infections occurred by chance rather than infection by one pathogen leading to an increased risk of contracting the other infection. However, co-infection risk was demonstrated in only two studies [26, 31], while other studies [27, 28, 32, 35] reported no difference in the odds of co-infection. The association between malaria and scrub typhus might be caused by the large geographic overlap in their distribution or the high prevalence of previous infections leading to cross-reactivity and subclinical infections rather than a high prevalence of co-infections. In addition, scrub typhus infections may prevent malaria or reduce its clinical presentation, but this has not yet been investigated, and further studies are needed.

The present study had limitations. First, only a small number of studies in Thailand and India reported malaria and scrub typhus co-infections. Therefore, the low prevalence of co-infection among febrile patients might be due to the underreporting of scrub typhus or misdiagnosis. Second, the high heterogeneity of measurements among the studies might have influenced the interpretation of the meta-analysis. Therefore, the pooled prevalence must be interpreted in consideration of the results of individual studies and sensitivity tests.

\section{Conclusion}

The findings of this study emphasize the importance of further research on malaria and scrub typhus co-infection in endemic areas. The outcomes also highlight the importance of interpreting diagnostic tests together with clinical signs and symptoms to facilitate the precise management of febrile patients who live in areas endemic for both diseases. 


\section{Abbreviations}

Cl: Confidence intervals; ELISA: Enzyme-linked immunosorbent assay; IFA: Indirect fluorescence assay; IQR: Interquartile range; NS: Not specified; PCR: Polymerase chain reaction; PRISMA: Preferred reporting criteria for systematic reviews and meta-analyses; RDT: Rapid diagnostic test; WHO: World Health Organization.

\section{Supplementary Information}

The online version contains supplementary material available at https://doi. org/10.1186/s13071-021-04969-y.

Additional file 1: Table S1. Search terms.

Additional file 2: Table S2. Quality of the included studies.

\section{Acknowledgements}

The authors would like to thank the New Strategic Research (P2P) project, Walailak University, Thailand, for providing partial funding for this study. We thank Suzanne Leech, Ph.D., from Liwen Bianji (Edanz) (www.liwenbianji.cn/), for basic language editing of a draft of this manuscript.

\section{Authors' contributions}

MK, SK, and PW carried out the study design, study selection, data extraction, and statistical analysis; and drafted the manuscript. PR reviewed and edited the manuscript. All authors read and approved the final manuscript.

\section{Funding}

This research was partially supported by the New Strategic Research (P2P) project, Walailak University, Thailand. The funders had a role in the collection, analysis, and interpretation of the data.

\section{Availability of data and materials}

All data relating to the present study in this manuscript are available.

\section{Declarations}

\section{Ethics approval and consent to participate}

Not applicable.

\section{Consent for publication}

Not applicable.

\section{Competing interests}

The authors declare that they have no competing interests.

\section{Author details}

'Department of Clinical Tropical Medicine, Faculty of Tropical Medicine, Mahidol University, Bangkok 10400, Thailand. ${ }^{2}$ Department of Medical Technology, School of Allied Health Sciences, Walailak University, Tha Sala, Nakhon Si Thammarat 80160, Thailand. ${ }^{3}$ Department of Protozoology, Faculty of Tropical Medicine, Mahidol University, Bangkok 10400, Thailand.

Received: 23 May 2021 Accepted: 18 Auqust 2021

Published online: 14 September 2021

\section{References}

1. Mahittikorn A, Masangkay FR, Kotepui KU, Milanez GJ, Kotepui M. Comparison of Plasmodium ovale curtisi and Plasmodium ovale wallikeri infections by a meta-analysis approach. Sci Rep. 2021;11:6409.

2. Brasil P, Zalis MG, de Pina-Costa A, Siqueira AM, Junior CB, Silva S, et al. Outbreak of human malaria caused by Plasmodium simium in the Atlantic Forest in Rio de Janeiro: a molecular epidemiological investigation. Lancet Glob Health. 2017;5:e1038-46.

3. Grigg MJ, Snounou G. Plasmodium simium: a Brazilian focus of anthropozoonotic vivax malaria? Lancet Glob Health. 2017;5:e961-2.
4. Hartmeyer GN, Stensvold CR, Fabricius T, Marmolin ES, Hoegh SV, Nielsen $\mathrm{HV}$, et al. Plasmodium cynomolgi as Cause of Malaria in Tourist to Southeast Asia, 2018. Emerg Infect Dis. 2019;25:1936-9.

5. Kotepui M, Masangkay FR, Kotepui KU, Milanez GJ. Preliminary review on the prevalence, proportion, geographical distribution, and characteristics of naturally acquired Plasmodium cynomolgi infection in mosquitoes, macaques, and humans: a systematic review and meta-analysis. BMC Infect Dis. 2021;21:259.

6. Cohee LM, Laufer MK. Malaria in Children. Pediatr Clin North Am. 2017;64:851-66.

7. World Health Organization. World malaria report 2020; 2020. https:// www.who.int/publications/i/item/9789240015791. Accessed 25 June 2021.

8. Mischlinger J, Ronnberg C, Alvarez-Martinez MJ, Buhler S, Paul M, Schlagenhauf $\mathrm{P}$, et al. Imported malaria in countries where malaria is not endemic: a comparison of semi-immune and nonimmune travelers. Clin Microbiol Rev. 2020;33:e00104-e119.

9. Luce-Fedrow A, Lehman ML, Kelly DJ, Mullins K, Maina AN, Stewart RL, et al. A review of scrub typhus (Orientia tsutsugamushi and related organisms): then, now, and tomorrow. Trop Med Infect Dis. 2018;3:8.

10. Varghese GM, Trowbridge P, Janardhanan J, Thomas K, Peter JV, Mathews $P$, et al. Clinical profile and improving mortality trend of scrub typhus in South India. Int J Infect Dis. 2014;23:39-43.

11. Kuo CC, Huang JL, Shu PY, Lee PL, Kelt DA, Wang HC. Cascading effect of economic globalization on human risks of scrub typhus and tick-borne rickettsial diseases. Ecol Appl. 2012;22:1803-16.

12. Abarca K, Martinez-Valdebenito C, Angulo J, Jiang J, Farris CM, Richards $\mathrm{AL}$, et al. Molecular description of a novel Orientia species causing scrub typhus in Chile. Emerg Infect Dis. 2020;26:2148-56.

13. Izzard L, Fuller A, Blacksell SD, Paris DH, Richards AL, Aukkanit N, et al. Isolation of a novel Orientia species (O. chuto. sp. nov.) from a patient infected in Dubai. J Clin Microbiol. 2010;48:4404-9.

14. Silva-Ramos CR, Jacinavicius FC, Weitzel T, Walker DH, Faccini-Martinez AA. Scrub typhus: a new cause of acute undifferentiated febrile illness in Latin America? Travel Med Infect Dis. 2021;43:102138.

15. Bonell A, Lubell Y, Newton PN, Crump JA, Paris DH. Estimating the burden of scrub typhus: a systematic review. PLoS Negl Trop Dis. 2017:11:e0005838.

16. Chao CC, Zhang Z, Belinskaya T, Chen HW, Ching WM. Leptospirosis and rickettsial diseases sero-conversion surveillance among U.S. military personnel in Honduras. Mil Med. 2021;43:usab120.

17. Kocher C, Jiang J, Morrison AC, Castillo R, Leguia M, Loyola S, et al. Serologic evidence of scrub typhus in the Peruvian Amazon. Emerg Infect Dis. 2017;23:1389-91.

18. Weitzel T, Martinez-Valdebenito C, Acosta-Jamett G, Jiang J, Richards AL, Abarca K. Scrub typhus in continental Chile, 2016-2018(1). Emerg Infect Dis. 2019;25:1214-7.

19. Rapsang AG, Bhattacharyya P. Scrub typhus. Indian J Anaesth. 2013:57:127-34.

20. Le Van N, Van Pham C, Nguyen Dang M, Van Dao T, Le TDQ, Vu Hoang H, et al. Clinical features, laboratory characteristics and prognostic factors of severity in patients with rickettsiaceae at two military hospitals. Northern Vietnam Infect Drug Resist. 2020;13:2129-38.

21. Liberati A, Altman DG, Tetzlaff J, Mulrow C, Gotzsche PC, loannidis JP, et al. The PRISMA statement for reporting systematic reviews and meta-analyses of studies that evaluate healthcare interventions: explanation and elaboration. BMJ. 2009;339:b2700.

22. Kotepui M, Kotepui KU, Milanez GD, Masangkay FR. Prevalence and proportion of Plasmodium spp. triple mixed infections compared with double mixed infections: a systematic review and meta-analysis. Malar J. 2020;19:224.

23. Kotepui KU, Kotepui M. Prevalence of and risk factors for Plasmodium spp. co-infection with hepatitis B virus: a systematic review and meta-analysis. Malar J. 2020;19:368.

24. Higgins JP, Altman DG, Gotzsche PC, Juni P, Moher D, Oxman AD, et al. The Cochrane Collaboration's tool for assessing risk of bias in randomised trials. BMJ. 2011;343:5928.

25. Higgins JPT, Thomas J, Chandler J, Cumpston M, Li T, Page MJ, Welch VA (editors). Cochrane Handbook for Systematic Reviews of Interventions version 6.2 (updated February 2021). 2021. https://training.cochrane.org/ handbook. Accessed 15 July 2021. 
26. Ahmad S, Dhar M, Mittal G, Bhat NK, Shirazi N, Kalra V, et al. A comparative hospital-based observational study of mono- and co-infections of malaria, dengue virus and scrub typhus causing acute undifferentiated fever. Eur J Clin Microbiol Infect Dis. 2016;35:705-11.

27. Bal M, Mohanta MP, Sahu S, Dwibedi B, Pati S, Ranjit M. Profile of pediatric scrub typhus in Odisha, India. Indian Pediatr. 2019;56:304-6.

28. Behera B, Biswal M, Das R, Dey A, Jena J, Dhal S, et al. Clinico-epidemiological analysis of scrub typhus in hospitalised patients presenting with acute undifferentiated febrile illness: a hospital-based study from Eastern India. Indian J Med Microbiol. 2019;37:278-80.

29. Chanyasanha C, Kaeburong K, Chenchittikul M, Sujirarat D. Seroprevalence of scrub typhus infection in patients with pyrexia at some malaria clinics in three western provinces of Thailand. Asian Pac J Allergy Immunol. 1998;16:119-25.

30. Mohanty L, Dhanawat A, Gupta P, Maheshwari G. Clinical manifestations and associated complications of scrub typhus in Odisha, India. J Clin Diagn Res. 2020;14:5.

31. Mørch K, Manoharan A, Chandy S, Chacko N, Alvarez-Uria G, Patil S, et al. Acute undifferentiated fever in India: a multicentre study of aetiology and diagnostic accuracy. BMC Infect Dis. 2017;17:665.

32. Raina S, Raina RK, Agarwala N, Raina SK, Sharma R. Coinfections as an aetiology of acute undifferentiated febrile illness among adult patients in the sub-Himalayan region of north India. J Vector Borne Dis. 2018;55:130-6.

33. Singh R, Singh SP, Ahmad N. A study of etiological pattern in an epidemic of acute febrile illness during monsoon in a tertiary health care institute of Uttarakhand, India. J Clin Diagn Res. 2014;8:Mc1-3.

34. SinghsilarakT, Phongtananant S, Jenjittikul M, Watt G, Tangpakdee N, Popak N, et al. Possible acute coinfections in Thai malaria patients. Southeast Asian J Trop Med Public Health. 2006;37:1-4

35. McGready R, Ashley EA, Wuthiekanun V, Tan SO, Pimanpanarak M, Viladpai-Nguen SJ, et al. Arthropod borne disease: the leading cause of fever in pregnancy on the Thai-Burmese border. PLoS Negl Trop Dis. 2010;4:10.

36. Kotepui M, PhunPhuech B, Phiwklam N, Uthaisar K, Thirarattanasunthon P. Prevalence of scrub typhus infection among patients infected with malaria in Phop Phra Hospital, Tak Province, Thailand. J Med Tech Assoc Thailand. 2017:45:5910-6.

37. Mandage R, Kaur C, Pramanik A, Kumar V, Kodan P, Singh A, et al. Association of dengue virus and leptospira co-infections with malaria severity. Emerg Infect Dis. 2020;26:1645-53.

38. Mittal GAS, Agarwal RK, Dhar M, Mittal M, Sharma S. Aetiologies of acute undifferentiated febrile illness in adult patients - an experience from a tertiary care hospital in Northern India. J Clin Diagn Res. 2015:9:DC22-4.

39. Patil R, Kulkarni S. Evaluation of acute febrile illness in patients presenting to a tertiary care hospital. Evolution Med Dent Sci. 2019;8:3801-4.

40. Willis $\mathrm{BH}$, Riley RD. Measuring the statistical validity of summary metaanalysis and meta-regression results for use in clinical practice. Stat Med. 2017:36:3283-301.

41. Rossati A, Bargiacchi O, Kroumova V, Zaramella M, Caputo A, Garavelli PL. Climate, environment and transmission of malaria. Infez Med. 2016;24:93-104

42. Chakraborty S, Sarma N. Scrub typhus: an emerging threat. Indian J Dermatol. 2017;62:478-85.
43. Kotepui M, Kotepui KU, Milanez GJ, Masangkay FR. Prevalence of and risk factors for severe malaria caused by Plasmodium and dengue virus co-infection: a systematic review and meta-analysis. Infect Dis Poverty. 2020;9:134

44. Rodkvamtook W, Kuttasingkee N, Linsuwanon P, Sudsawat Y, Richards AL, Somsri M, et al. Scrub Typhus outbreak in Chonburi Province, Central Thailand, 2013. Emerg Infect Dis. 2018;24:361-5.

45. Kim YJ, Park S, Premaratna R, Selvaraj S, Park SJ, Kim S, et al. Clinical evaluation of rapid diagnostic test kit for scrub typhus with improved performance. J Korean Med Sci. 2016;31:1190-6.

46. Saraswati K, Day NPJ, Mukaka M, Blacksell SD. Scrub typhus point-ofcare testing: a systematic review and meta-analysis. PLoS Negl Trop Dis. 2018;12:e0006330.

47. Varghese GM, Abraham OC, Mathai D, Thomas K, Aaron R, Kavitha ML, et al. Scrub typhus among hospitalised patients with febrile illness in South India: magnitude and clinical predictors. J Infect. 2006;52:56-60.

48. Sinha P, Gupta S, Dawra R, Rijhawan P. Recent outbreak of scrub typhus in North Western part of India. Indian J Med Microbiol. 2014;32:247-50.

49. Nadjm B, Thuy PT, Trang VD, le Ha D, Kinh NV, Wertheim HF. Scrub typhus in the northern provinces of Vietnam: an observational study of admissions to a national referral hospital. Trans R Soc Trop Med Hyge. 2014;108:739-40.

50. Trung NV, Hoi LT, Thuong NTH, Toan TK, Huong TTK, Hoa TM, et al. Seroprevalence of scrub typhus, typhus, and spotted fever among rural and urban populations of Northern Vietnam. Am J Trop Med Hyg. 2017;96:1084-7.

51. Tshokey T, Stenos J, Durrheim DN, Eastwood K, Nguyen C, Graves SR. Seroprevalence of rickettsial infections and Q fever in Bhutan. PLoS Negl Trop Dis. 2017;11:e0006107.

52. Tshokey T, Stenos J, Durrheim DN, Eastwood K, Nguyen C, Vincent G, et al. Rickettsial infections and Q fever amongst febrile patients in Bhutan. Trop Med Infect Dis. 2018;3(1):12.

53. Tay ST, Kamalanathan M, Rohani MY. Antibody prevalence of Orientia tsutsugamushi, Rickettsia typhi and TT118 spotted fever group rickettsiae among Malaysian blood donors and febrile patients in the urban areas. Southeast Asian J Trop Med Public Health. 2003;34:165-70.

54. Tay ST, Ho TM, Rohani MY, Devi S. Antibodies to Orientia tsutsugamushi, Rickettsia typhi and spotted fever group rickettsiae among febrile patients in rural areas of Malaysia. Trans R Soc Trop Med Hyg. 2000;94:280-4.

55. Richards AL, Jiang J. Scrub typhus: historic perspective and current status of the worldwide presence of Orientia species. Trop Med Infect Dis. 2020;5:49.

56. Xu G, Walker DH, Jupiter D, Melby PC, Arcari CM. A review of the global epidemiology of scrub typhus. PLoS Negl Trop Dis. 2017;11:e0006062.

57. Hanefeld J. The Global fund to fight aids, tuberculosis and malaria: 10 years on. Clin Med (Lond). 2014;14:54-7.

\section{Publisher's Note}

Springer Nature remains neutral with regard to jurisdictional claims in published maps and institutional affiliations.

Ready to submit your research? Choose BMC and benefit from

- fast, convenient online submission

- thorough peer review by experienced researchers in your field

- rapid publication on acceptance

- support for research data, including large and complex data types

- gold Open Access which fosters wider collaboration and increased citations

- maximum visibility for your research: over $100 \mathrm{M}$ website views per year

At $\mathrm{BMC}$, research is always in progress.

Learn more biomedcentral.com/submissions 